\title{
Les résidus non extractibles de produits phytosanitaires dans les sols
}

\author{
G. Bertin et M. Schiavon \\ ENSAIA, Service Protection des Cultures, BP 172, 2, av. de la forêt de Haye, 54505 Vandœuvre-lès-Nancy Cedex, France
}

(reçu le 4-5-1988, accepté le 8-11-1988)

\begin{abstract}
Résumé - Les résidus non extractibles formés par les produits phytosanitaires ne sont pas détectables par les méthodes d'analyse conventionnelles, et leur existence dans les sols n'est connue que depuis l'utilisation des molécules marquées. La formation de résidus non extractibles a été mise en évidence pour toutes les classes de produits phytosanitaires à des degrés différents, mais leur identification chimique n'a été réalisée que pour un nombre limité de substances. Dans la plupart des cas, ces résidus ne sont pas associés à une seule fraction du sol, mais la matière organique semble jouer un rôle prépondérant. Sensibles aux attaques microbiennes, leur biodisponibilité vis-à-vis des plantes et de la faune du sol a été démontrée.
\end{abstract}

résidus non extractibles - produit phytosanitaire - sol - méthodes d'analyse - biodisponibilité

Summary - Non-extractable pesticide residues in soils. The non-extractable pesticide residues escape detection by the conventional analytical methods and their existence in soils has been known since the use of radiolabeled pesticides. Non-extractable residues have been detected for all classes of pesticides investigated with different quantitative levels, but their chemical identity has only been elucidated for a limited number of substances. In most cases, the formation of non-extractable residues cannot be correlated to a unique soil fraction but the organic matter appears to play the most important role. Finally, the non-extractable pesticide residues seem to be susceptible to microbial attack and their bioavailability to plants and soil fauna has been demonstrated.

non-extractable residues - pesticide - soil - analytical methods - bioavailability

\section{Introduction}

Certaines molécules organiques de synthèse, utilisées pour la protection des cultures, peuvent, lorsqu'elles sont appliquées en traitement de sol, former avec ses constituants des liaisons irréversibles et conduire à la formation de ce qui est appelé des «résidus non extractibles».

En 1975, I'U.S. Environmental Protection Agency définit ainsi ces résidus : "Résidus de pesticides non extractibles par les solvants organiques, non identifiables chimiquement et qui restent, après extraction exhaustive, au sein des fractions "acides fulviques", "acides humiques" et "humine".

L'expression "résidus non extractibles» englobe les résidus de produits xénobiotiques véritablement liés aux constituants du sol par liaison covalente, et ceux, également non extractibles, simplement retenus par d'autres mécanismes, que les techniques d'extraction communément employées ne permettent pas de recouvrer.

C'est grâce à l'utilisation de molécules marquées par un isotope radioactif que ce phénomène a été mis en évidence. Les travaux menés depuis une dizaine d'années sur des produits très divers, de par leur nature chimique et leurs propriétés physiques, tendent à montrer que la formation de résidus non extractibles intéresse toutes les familles chimiques de produits phytosanitaires.

Les valeurs concernant l'importance quantitative du phénomène sont difficilement comparables. Elles varient, en pourcentage de la dose appliquée, entre $7 \%$ pour la dieldrine, après une période de contact sol-produit de 28 jours (Lich- 
tenstein et al., 1977), et $90 \%$ pour la dichloroaniline (Viswanathan et al., 1978), mais dans ce cas l'interaction avec les constituants du sol est de 18 mois.

Il est démontré, d'une manière générale, que la liaison de ces molécules se fait principalement avec les composants organiques du sol. Ainsi, Ambrosi et al., (1977), étudiant le comportement de l'oxadiazon dans deux types de sol, ont mis en évidence une relation entre le taux de matière organique et le pourcentage de résidus non extractibles formés. Cependant, certains auteurs n'excluent pas la formation de liaisons irréversibles avec la fraction minérale (Helling et Krivonak, 1978a).

Différentes hypothèses sont avancées sur la nature de ces liaisons sol-produit phytosanitaire, sur leur stabilité et sur le rôle joué par les différentes fractions humiques dans la formation des résidus non extractibles. Nous nous proposons de faire le point sur les différents aspects concernant ces recherches.

\section{Etat des connaissances}

\section{Méthodes d'étude}

La procédure «classique», qui diffère quelque peu suivant les chercheurs, consiste à traiter un sol, au laboratoire ou au champ, avec un pesticide marqué au ${ }^{14} \mathrm{C}$. A intervalles de temps réguliers, un échantillon est prélevé et soumis à une extraction exhaustive. Le solvant est choisi en fonction de son efficience dans la procédure d'extraction du produit phytosanitaire considéré. Les techniques utilisées sont nombreuses, mais l'une des plus employées est celle faisant appel à l'appareil de Soxhlet, bien que certains auteurs indiquent l'obtention d'un meilleur rendement par agitation mécanique du sol en présence du solvant à froid.

Lorsque l'extrait est dépourvu de toute radioactivité, on considère que celle subsistant dans le sol est non extractible.

II faut noter que les résultats obtenus peuvent être très variables suivant la procédure d'extraction adoptée. Tant qu'il n'existera pas de méthode performante standardisée, il conviendra d'être prudent dans la comparaison des résultats obtenus par différents auteurs.

Après extraction exhaustive, l'évaluation des résidus non extractibles formés est obtenue par combustion du sol et mesure du ${ }^{14} \mathrm{CO}_{2}$ libéré. Cela permet une quantification du phénomène, mais ne donne aucune indication sur le mode de liaison, sur les constituants du sol mis en cause, sur les facteurs qui influent sur le ou les méca- nismes de formation, et sur la nature chimique du ou des produits non extractibles. Aussi certains chercheurs ont mis en œuvre d'autres méthodes d'étude.

\section{Méthodes hydrolytiques}

Après extraction exhaustive du sol, Hsu et Bartha (1976) procèdent à l'hydrolyse alcaline d'un échantillon soumis préalablement à un traitement à la 3,4-dichloroaniline. Ils distinguent ainsi, parmi les complexes humus-3,4-dichloroaniline, une fraction hydrolysable et une fraction non hydrolysable au sein desquelles la liaison produit xénobiotique-matière organique pourrait être différente. Dans un sol traité à l'oryzaline ${ }^{14} \mathrm{C}$, Golab et al. (1975), constatent que $30 \%$ de la radioactivité initialement introduite devient non extractible. Dans ce cas, une hydrolyse par $\mathrm{KOH}$ $0,5 \mathrm{~N}$, pendant $48 \mathrm{~h}$, permet la libération de $90 \%$ de cette radioactivité. Cependant les produits obtenus sont altérés et leur identification s'avère impossible.

Pour Worobey et Webster (1982a), l'hydrolyse acide $(\mathrm{HCl} 6 \mathrm{~N})$ ou alcaline $(\mathrm{NaOH} 0,5 \mathrm{~N})$, conduit au même résultat. Ils obtiennent dans chacun des cas, la libération de $90 \%$ de la radioactivité fixée sur les acides humiques après traitement à la 4-chloroaniline. Dans leur expérimentation, plus de la moitié de la radioactivité est identifiée comme étant la molécule mère. Les méthodes hydrolytiques apparaissent donc peu satisfaisantes et la généralisation de leur utilisation dans cette démarche n'est pas envisageable.

\section{Méthodes pyrolytiques}

Pour identifier les résidus non extractibles de prométryne, Khan et Hamilton (1980) ont utilisé une méthode de distillation des composés organiques du sol à haute température, sous hélium.

Les résidus libérés sont collectés dans des solvants appropriés et analysés par chromatographie en phase gazeuse et spectrométrie de masse. Malgré une décomposition thermique des résidus au cours de la distillation, le taux de radioactivité récupérée et identifiée atteint $80 \%$ du total des résidus non extractibles.

Par contre, Worobey et Webster (1982b) en soumettant leurs échantillons à une pyrolyse, ne récupèrent que $54 \%$ de la radioactivité non extractible formée à la suite d'un traitement à la 4-chloroaniline.

Ici encore, I'utilisation de températures élevées conduit à une dégradation. Celle-ci paraît plus ou moins importante en fonction de la stabilité de la molécule utilisée.

II apparaît donc que les méthodes hydroly- 
tiques ou pyrolytiques sont deux techniques permettant un progrès des connaissances, mais à utiliser d'une manière complémentaire après avoir établi dans chaque situation la stabilité du produit considéré.

\section{Cinétique de formation des résidus non extrac- tibles}

A partir d'un sol extrait de manière exhaustive, on peut doser le ${ }^{14} \mathrm{CO}_{2}$ libéré par combustion et calculer, par différence, la quantité totale de résidus non extractibles formés.

Lors d'une incubation d'un sol avec divers insecticides marqués (parathion, fonofos, dieldrine, DDT), Lichtenstein et al. (1977), procèdent à des prélèvements et des dosages de ${ }^{14} \mathrm{CO}_{2}$ à intervalles réguliers pendant un mois. Ils constatent ainsi que la quantité de résidus non extractibles augmente parallèment à une décroissance des résidus totaux et des résidus extractibles, jusqu'à l'obtention d'un palier.

Avec le même type d'expérimentation, mais prolongée sur plusieurs années, Klein et Scheunert (1982) constatent que le taux de résidus non extractibles demeure stable, malgré une décroissance marquée des résidus totaux. Tout se passe comme si la formation de résidus non extractibles à partir de la molécule mère ou de ses métabolites était compensée par une lente minéralisation (Tableau I).

Ainsi le sol paraît exercer un pouvoir épurateur qui se décompose en deux étapes : une première phase de stockage après traitement suivie par l'élimination progressive de ces résidus par minéralisation, au même titre que la matière organique endogène.
Fractions du sol impliquées dans la formation de résidus non extractibles

Après avoir examiné l'aspect quantitatif de la formation de résidus non extractibles, certains chercheurs se sont intéressés à leur localisation au sein des différentes fractions du sol.

L'utilisation de la méthode «classique» d'extraction et de fractionnement de la matière organique permet à Helling et Krivonak (1978b) de préciser que la majeure partie des résidus non extractibles issus de la dinitroaniline (50-60\%) est associée aux acides fulviques sauf en conditions anaérobies où l'humine $(46 \%)$ et les acides humiques $(40 \%)$ constituent alors les fractions les plus riches.

Spillner et al. (1979), lors d'une étude de la dégradation du fenitrothion en sol forestier, trouvent au contraire que la plus grande partie des résidus non extractibles est fixée aux acides humiques $(30-44 \%)$ puis à l'humine $(20-30 \%)$ et seulement $12-34 \%$, aux acides fulviques. Une toute autre distribution des résidus non extractibles est observée par Khan (1982) avec un sol organique traité à la prométryne. Après un an d'incubation, la proportion de résidus non extractibles dans les fractions humine, acides humiques et acides fulviques est respectivement de 57,10 , et $26 \%$.

Schiavon et al. (1977), montrent que le pourcentage de résidus non extractibles bloqués respectivement sur chaque fraction de la matière organique évolue rapidement avec le temps. Au cours d'une incubation de 31 jours, on observe une diminution du pourcentage de radioactivité résiduelle fixée sur les acides fulviques au profit des fractions plus humifiées (Tableau II).

Tableau I. Résidus non extractibles et résidus totaux de ${ }^{14} \mathrm{C}$ aldrine dans un sol durant 10 ans après l'application.

\begin{tabular}{|c|c|c|}
\hline Année & $\begin{array}{l}\text { Résidus non } \\
\text { extractibles }\end{array}$ & $\begin{array}{l}\text { Résidus } \\
\text { totaux }\end{array}$ \\
\hline 1969, après application & 0 & 2,72 \\
\hline 1969 , après récolte & 0,17 & 1,87 \\
\hline 1970 & 0,11 & 1,18 \\
\hline 1971 & 0,06 & 0,57 \\
\hline 1972 & 0,13 & 0,64 \\
\hline 1973 & 0,11 & 0,50 \\
\hline 1974 & 0,09 & 0,44 \\
\hline 1975 & 0,11 & 0,43 \\
\hline 1979 & 0,11 & 0,35 \\
\hline
\end{tabular}

Profondeur $0-10 \mathrm{~cm}$, conditions extérieures. 
Tableau II. Répartition (en \%) des résidus non extractibles d'atrazine dans les fractions de la matière organique du sol.

\begin{tabular}{llll}
\hline $\begin{array}{l}\text { Durée de } \\
\text { l'incubation (jours) }\end{array}$ & $\begin{array}{l}\text { Acides } \\
\text { fulviques }\end{array}$ & $\begin{array}{l}\text { Acides } \\
\text { humiques }\end{array}$ & Humine \\
\hline 2 & 77,0 & & 23 \\
4 & 57,6 & 11,9 & 30,5 \\
12 & 54,3 & 12,5 & 33,2 \\
31 & 47,7 & 13,9 & 38,4 \\
\hline
\end{tabular}

Mais Schiavon et Soulas (1983), montrent que l'évaluation des résidus non extractibles formés est étroitement dépendante des performances des solvants et des techniques d'extraction. Utilisant l'atrazine, ils observent que lorsqu'on effectue une série d'extractions successives, l'extraction complémentaire de radioactivité se fait essentiellement aux dépens de celle fixée sur les acides fulviques. Cela amène les auteurs à différencier deux catégories de résidus inextractibles : les "résiduels" fixés aux acides fulviques, dont la présence dans la fraction «résidus non extractibles" serait pour partie consécutive à des difficultés techniques d'extraction, et les "stables", fixés aux acides humiques et à l'humine.

Si la majeure partie des auteurs ont pu mettre en évidence le rôle de la matière organique dans la formation de résidus noh extractibles, Capriel et al. (1985) ont montré que la fraction minérale pouvait également participer à ce processus.

Neuf ans après avoir traité un sol avec de l'atrazine marquée, et sous conditions de plein champ, la moitié de la radioactivité encore présente au moment du prélèvement des échantillons se trouve sous forme inextractible. Après fractionnement de la matière organique, les auteurs indiquent que $44 \%$ des résidus sont associés à l'humine, $33 \%$ aux acides fulviques, $13 \%$ aux acides humiques et $10 \%$ reste localisé au sein des composés minéraux du sol.

- Cette revue des données obtenues par différents auteurs montre une très grande diversité et même certaines contradictions quant à la localisation des résidus non extractibles dans les différentes fractions du sol.

II ne faut cependant pas perdre de vue que les molécules et les situations examinées ne sont jamais identiques. Par ailleurs, l'extraction alcaline de la matière organique, puis son fractionnement à $\mathrm{pH}$ acide, offrent des conditions favorables aux réactions d'hydrolyse, d'oxydation et de condensation, susceptibles de perturber la répartition initiale des résidus non extractibles.

\section{Nature chimique des résidus non extractibles}

Dans une nouvelle définition, publiée en 1984, I'International Union of Pure and Applied Chemistry (IUPAC) considère que doivent être exclus de la fraction "résidus non extractibles» les fragments de molécule pesticide recyclés par des voies métaboliques et conduisant à des produits naturels. Dans ces conditions, l'évaluation de la radioactivité non extractible ne rend pas compte de l'importance de ces résidus. Il est donc nécessaire d'identifier la part de cette radioactivité revenant à la molécule mère et / ou à ses métabolites.

Au cours d'études sur le comportement de la trifluraline dans le sol, Golab et al. (1979) mettent en évidence 28 produits de dégradation, dont aucun n'occupe une place prépondérante.

A partir d'une incubation de sol traité à la prométryne Khan et Hamilton (1980) indiquent qu'après deux mois la molécule mère et ses métabolites déalkylés représentent $54 \%$ de la radioactivité non extractible.

Schiavon (1988) montre que l'atrazine et tous ses métabolites chlorés et hydroxylés sont susceptibles de contracter des liaisons irréversibles avec le sol mais le phénomène est très discret avec l'hydroxy-atrazine. S'intéressant également à l'atrazine, Capriel et al. (1985) soulignent que, neuf ans après l'application de l'herbicide, les composés dominants au sein de la fraction non extractible sont la molécule mère et ses métabolites hydroxylés. Enfin, Scheunert et al. (1985) se sont intéressés à la relation existant entre la structure moléculaire du pesticide et les possibilités de formation de résidus non extractibles. Avec une série de chlorobenzènes et des conditions expérimentales proches de celles de plein champ, les auteurs montrent que les taux de résidus non extractibles formés sont corrélés négativement avec le nombre d'atomes de chlore présents sur la molécule pesticide.

Ces quelques exemples illustrent l'extrême variété des situations qui sont à mettre en rela- 
tion avec la diversité des molécules phytosanitaires existantes.

Ainsi, il semblerait que certains produits se lient aux constituants du sol de manière irréversible sans aucune transformation préalable, alors que d'autres doivent subir une dégradation qui les conduit à une forme plus réactive. A ce propos, Katan (1976) a montré que la liaison du parathion est subordonnée à la présence de microorganismes dans le sol, qui réduisent cet insecticide en composé amino, alors qu'il n'en est pas de même pour le fonofos.

De même, Venkateswarlu et Sethunathan (1979), dans une étude concernant le métabolisme du carbofuran dans le sol, ont constaté que des conditions favorables à l'hydrolyse du carbofuran en carbofuran phénol conduisaient à une augmentation significative du taux de résidus non extractibles.

\section{Nature des liaisons}

Les résidus facilement extractibles sont fixés au sol par des liaisons de faible énergie. On parle alors de phénomène d'adsorption. Par contre, les résidus non extractibles doivent nécessairement contracter avec le complexe organo-minéral des liaisons plus fortes, dont la nature exacte reste hypothétique. A cet égard, des études portant sur des composés synthétiques, mieux définis que les fractions organiques du sol et parfaitement reproductibles apportent quelques éléments de réponse.

Selon Hsu et Bartha (1976), le fait que les résidus non extractibles résistent aux extractions organiques et aux échanges d'ions mais soient sensibles aux hydrolyses acides et alcalines, suggère l'existence d'une liaison covalente. Les résultats obtenus lors de la synthèse d'un modèle humique à partir de méthylcatéchol et de dichloroaniline, permettent à ces auteurs d'avancer l'hypothèse d'une incorporation du pesticide à l'intérieur d'un système d'hétérocycles.

Par contre, les résultats obtenus par Smith et al. (1973) ne soutiennent pas cette hypothèse. En effet, le traitement par la soude $0,25 \mathrm{~N}$ d'un sol contenant des résidus non extractibles de dinitramine aboutit à la libération d'environ 50\% de la radioactivité non extractible, tandis que l'acide chlorhydrique concentré s'avère bien moins efficient. Pour ces auteurs les résidus ne sont donc pas liés par covalence, mais simplement «fortement» adsorbés.

Une autre hypothèse est avancée par Schiavon et al. (1977). Ces résidus pourraient être, au moins pour partie, emprisonnés au sein du réseau tridimensionnel des macromolécules humiques. C'est également l'opinion exprimée par Khan (1982) qui pense qu'en plus des liaisons chimiques, les pesticides ou leurs métabolites peuvent être retenus à l'intérieur des lacunes observées dans la structure des polymères humiques.

La synthèse de modèles humiques en présence d'un pesticide permet une autre approche du problème, et apporte quelques informations, qui dans une première analyse paraissent contradictoires. Ainsi You et al. (1982) réalisent la polymérisation du méthylcatéchol en présence de 3,4-dichloroaniline. Le produit de cette interaction est isolé, analysé puis soumis à des hydrolyses acides ou alcalines, et à une biodégradation. Considérant les résultats obtenus, les auteurs suggèrent la formation d'une liaison covalente entre le méthylcatéchol et la 3,4dichloroaniline au niveau du groupement aminé du pesticide. Cependant la possibilité de formation de cette liaison n'est pas généralisable, car la sensibilité de ce modèle aux hydrolyses et à la biodégradation est différente de celle des complexes dichloroaniline - humus.

Mais le remplacement du méthylcatéchol par la toluquinone dans ce type de réaction modélisée conduit à des résultats sensiblement différents. La sensibilité à l'hydrolyse acide du complexe obtenu est dans ce cas similaire à celle du complexe 3,4-dichloroaniline - humus. II demeure toutefois moins sensible à l'hydrolyse alcaline. Les auteurs (Saxena et Bartha, 1983a) considèrent que cette différence de comportement du modèle par rapport au complexe 3,4-dichloroaniline - humus peut être attribuée à sa structure beaucoup plus simple, bien que les possibilités de biodégradation des deux systèmes soient identiques. Saxena et Bartha (1983a) rejettent I'hypothèse de You et al. (1982), qui considèrent qu'on ne peut généraliser les résultats obtenus à l'aide d'un modèle, et évoquent l'existence d'une liaison au niveau des sites quinoniques des acides humiques.

Les travaux conduits par Bollag et al. (1983) vont dans le même sens. En faisant réagir trois anilines (4-chloroaniline, 3,4-dichloroaniline, 2,6-diéthylaniline) avec différents constituants phénoliques de l'humus en présence d'une oxydase fongique, produite par Rhizoctonia praticola, les auteurs isolent différents composés dimères, trimères et tétramères, dont la structure est déterminée par spectrométrie de masse. Ils constatent que dans ces conditions, les molécules phénoliques sont préalablement oxydées en quinones, qui réagissent avec les anilines en contractant une liaison entre l'atome d'azote et un atome de carbone du noyau aromatique, formant ainsi une fonction imine. Ces composés 
seraient ensuite rapidement incorporés dans la matière organique.

En fait, la formation de résidus non extractibles semble dépendre de processus différents suivant les espèces chimiques mises en jeu et les conditions de milieu. En effet, à l'intérieur de la fraction "résidus non extractibles" coexistent une partie stabilisée et une partie susceptible d'être libérée par l'intervention de phénomènes physiques ou biologiques.

\section{La libération des résidus non extractibles}

L'existence d'une possibilité de libération des résidus non extractibles constitue une préoccupation essentielle tant du point de vue agronomique qu'écologique. Cette possibilité de libération a été mise en évidence par Khan et Ivarson (1981) avec la prométryne. Après 22 jours d'incubation, $27 \%$ de la radioactivité non extractible est recouvrée, dont la moitié est identifiée comme étant la molécule mère.

Il semble donc que les microorganismes soient susceptibles de rompre les liaisons établies entre l'herbicide et les constituants du sol, mais il n'a pas été possible de définir le type de populations mises en cause. En effet, le même type d'incubation est renouvelé par Khan et Ivarson (1982) en sélectionnant quatre souches différentes de microorganismes spécifiques de la dégradation de la cellulose, des protides, des lipides et de la lignine. Ils constatent ainsi que la nature de la population microbienne n'influe pas significativement sur la quantité des résidus libérés. Pour les auteurs, ce résultat serait dû au fait que les processus de libération impliquent un phénomène de cométabolisme, et/ou que les résidus non extractibles sont inclus dans différentes matrices.

L'activité microbienne ne constitue pas le seul facteur pouvant aboutir à la libération des résidus non extractibles. Khan (1982) remarque que la stabilité des résidus non extractibles de prométryne dans une suspension de sol est affectée par les rayonnements ultra-violets. Après une exposition aux UV de $216 \mathrm{~h}, 30 \%$ des résidus deviennent extractibles par le méthanol. La majeure partie de ceux-ci se trouve sous forme hydroxylée, mais l'hydroxylation, induite par les UV, est postérieure à la libération des résidus.

Des modifications de la structure physique du sol, à la suite d'une dessication, peuvent également entraîner une libération importante de radioactivité non extractible. Schiavon et Soulas (1983) observent que ce phénomène est particulièrement marqué lorsque cette radioactivité a pour origine le traitement du sol par deux métabolites déalkylés de l'atrazine : le dééthyl-déiso- propyl atrazine et le dééthyl atrazine. Cette libération serait due à une modification de la distribution spatiale des chaînes aliphatiques des molécules humiques, primitivement à l'origine de l'emprisonnement des molécules pesticides.

Parmi les travaux portant sur la recherche des différents facteurs pouvant conduire à la libération des résidus non extractibles, l'étude de l'incidence de la fertilisation et des modifications du $\mathrm{pH}$ du sol a été également abordée. Saxena et Bartha (1983b) ont pu remarquer que les complexes 3,4-dichloroaniline-humus étaient sensibles à un traitement par $\mathrm{NH}_{4} \mathrm{OH}$. Après une incubation de 30 jours, $11 \%$ des résidus non extractibles sont libérés. Les auteurs indiquent donc que la fertilisation azotée sous forme ammoniacale est susceptible d'aboutir à la mobilisation de quantités significatives de résidus non extractibles d'aniline.

Ce même type d'expérimentation a été repris et complété par Yee et al. (1985), qui ont étudié l'effet de trois sources d'azote sur la libération des résidus non extractibles de prométryne. D'après leurs résultats il apparaît que ce sont les formes ioniques $\mathrm{NH}_{4}^{+}$et $\mathrm{NO}_{3}^{-}$qui libèrent le plus de résidus non extractibles, tandis que l'urée apparaît pratiquement sans effet.

Ces mêmes auteurs se sont également intéressés aux conséquences de la modification du $\mathrm{pH}$ du sol. Pour cela des échantillons d'un même sol contenant des résidus non extractibles sont mis en présence de solutions tampon, dans une gamme de $\mathrm{pH}$ comprise entre 4 et 8 . Dans ces conditions, la radioactivité libérée varie entre 21 et $25 \%$ (en pourcentage du total non extractible), excepté à pH 5 où elle n'atteint que $15 \%$. Parmi les résidus extraits, la prométryne représente $70 \%$ et l'hydroxyprométryne $30 \%$ du total, excepté à $\mathrm{pH} 5$ où la part de chacun de ces deux produits est respectivement de 46 et $54 \%$.

Cet ensemble de résultats tend à montrer que des changements de $\mathrm{pH}$ et des interactions ioniques peuvent déplacer les résidus non extractibles, en modifiant la répartition des charges de la molécule pesticide et la configuration des polymères humiques.

Ainsi, un nombre important de facteurs paraît pouvoir intervenir sur la libération des résidus non extractibles. Un travail considérable reste à faire pour les définir et apprécier leur rôle respectif, probablement variable en fonction du produit phytosanitaire considéré.

Absorption des résidus non extractibles par les plantes et les organismes de la faune du sol

Les travaux que nous venons de présenter mettent en évidence deux faits essentiels : 1) Une 
partie au moins de la radioactivité non extractible peut être libérée sous l'action de nombreux facteurs (activité microbienne, dessication du sol, modification du $\mathrm{pH}$, fertilisation, etc.); 2) Parmi les résidus non extractibles susceptibles d'être libérés, coexiste avec les métabolites une part plus ou moins importante constituée par la molécule mère. Ces deux points étant démontrés, il restait à vérifier si cette libération possible pouvait avoir une conséquence significative tant sur le plan écologique qu'agronomique.

Les différents travaux menés en vue de répondre à ces questions montrent que les résidus non extractibles peuvent passer dans la plante et que dans certains cas on peut observer des effets phytotoxiques. Ainsi Fuhremann et Lichtenstein (1978), en implantant une culture d'avoine sur un sol contenant des résidus non extractibles de parathion, observent qu'après deux semaines les plantes ont absorbé une partie de l'insecticide.

Avec un sol contenant des résidus non extractibles de prométryne, Khan (1980) obtient des résultats semblables. Au cours de son expérimentation, l'avoine absorbe $0,53 \%$ de la radioactivité non extractible dont $70 \%$ se retrouve dans les parties aériennes et $30 \%$ dans les racines. Une partie de cette radioactivité absorbée par la plante demeure non extractible et est majoritairement associée à la lignine.

Pour leur part, Mostafa et al. (1982) font pousser des plants de haricot sur plusieurs types de sols contenant des résidus non extractibles de trifluraline, différant par leur texture ou par l'intervention d'un autoclavage préalable. Après un mois de croissance, les auteurs mesurent des concentrations variables d'herbicide dans le végétal, et de plus observent un léger retard de croissance et quelques déformations des feuilles pour les plantes ayant poussé sur sol sableux autoclavé. Des phénomènes de phytotoxicité ont été également signalés par Helling et Krivonak (1978b), lors d'une étude de l'absorption de résidus non extractibles de dinitroaniline, mais les auteurs attribuent ces symptômes aux procédures d'extraction des pesticides, qui en détruisant les microorganismes du sol affectent ainsi les possibilités de croissance ultérieure des végétaux.

Ainsi donc, utilisée comme système révélateur, la plante confirme une possibilité de mobilisation des résidus non extractibles avec accessoirement l'apparition de signes de phytotoxicité. Cette libération des résidus non extractibles affecte donc les végétaux, mais aussi certains organismes ayant des rapports trophiques avec le sol. Ainsi, plusieurs études font état de la disponibilité des résidus non extractibles vis-à-vis de la faune du sol et particulièrement des vers de terre (Fuhremann et Lichtenstein, 1978; Haque et al., 1982). Cette diversité concernant le devenir des résidus non extractibles après leur libération apparaît comme tout à fait normale, car leur comportement est alors celui d'une molécule libre. On constatera cependant que cet effet contaminant secondaire ne peut être que limité car il est tributaire de la cinétique de libération des résidus non extractibles.

\section{Conclusion}

La notion même de "résidus non extractibles" résulte de l'inefficacité des procédures d'extraction employées à rompre toutes les liaisons que les produits phytosanitaires, ou leurs métabolites, contractent avec les constituants du sol au cours du temps.

Même si dans la quantification de ces résidus subsiste une part relevant de l'artéfact provoqué par l'inadaptation des techniques d'extraction et de fractionnement, on peut supposer que la majeure partie des produits regroupés sous cette expression générique a contracté des liaisons suffisamment fortes pour entraîner une accumulation, plus ou moins irréversible au sein de l'écosystème sol.

A l'heure actuelle, nos connaissances, bien qu'importantes, sont encore fragmentaires. Pour quelques produits, on dispose d'informations sur la localisation, la nature et la disponibilité des résidus non extractibles. II n'en demeure pas moins qu'il convient de poursuivre les recherches pour comprendre les mécanismes de formation, de libération et préciser les facteurs biologiques, chimiques et physiques qui les conditionnent.

\section{Références}

Ambrosi D., Kearney P.C. \& Macchia J.A., (1977) Persistence and metabolism of oxadiazon in soils. $J$. Agric. Food Chem. 25, 868-872

Bollag J.M., Minard R.D. \& Liu S.Y., (1983) Cross-linkage between anilines and phenolic humus constituents. Environ. Sci. Technol. 17, 72-80

Capriel P., Haisch A. \& Khan S.U. (1985) Distribution and nature of bound (non-extractable) residues of atrazine in a mineral soil nine years after the herbicide application. J. Agric. Food Chem. 33, 567-569

Fuhremann W. \& Lichtenstein E.P., (1978) Release of soil bound methyl ${ }^{14} \mathrm{C}$ parathion residues and their uptake by earthworms and oat plants. J. Agric. Food Chem. 26, 605-610

Golab T., Bishop C.E., Donoho A.L., Manthey J.A. \& Zornes L.L. (1975) Behavior of ${ }^{14} \mathrm{C}$ oryzalin in soil and plants. Pestic. Biochem. Physiol. 5, 196-204

Golab T., Althaus W.A. \& Wooten H.L., (1979) Fate of 
${ }^{14} \mathrm{C}$ trifluralin in soil. J. Agric. Food Chem. 27, 163-179 Haque A., Schuphan I. \& Ebing W. (1982) Bioavailability of conjugated and soil bound ${ }^{14} \mathrm{C}$ hydroxymono-linuron-D-glucoside residues to earthworms and ryegrass. Pestic. Sci. 13, 219-228

Helling C.S. \& Krivonak A.E. (1978a) Physicochemical characteristics of bound dinitroaniline herbicides in soils. J. Agric. Food Chem. 26, 1156-1163

Helling C.S. \& Krivonak A.E., (1978b) Biological characteristics of bound dinitroaniline herbicides in soils. J. Agric. Food Chem. 26, 1164-1172

Hsu T.S. \& Bartha R. (1976) Hydrolysable and non hydrolysable 3,4-dichloroaniline - humus complexes and their respective rates of biodegradation. J. Agric. Food. Chem. 24, 118-122

IUPAC Reports on Pesticides (1984) Non-extractable pesticide residues in soils and plants. Pure Appl. Chem. 56, 945-956

Katan I. (1976) Binding of ${ }^{14} \mathrm{C}$ parathion in soil: a reassessment of pesticide persistence. Science 193-891

Khan S.U. (1980) Plant uptake of unextracted (bound) residues from an organic soil treated with prometryn. J. Agric. Food Chem. 28, 1096-1098

Khan S.U. (1982) Distribution and characteristics of bound residues of prometryn in an organic soil. $J$. Agric. Food Chem. 30, 175-179

Khan S.U. \& Hamilton H.A., (1980) Extractable and bound (nonextractable) residues of prometryn and its metabolites in an organic soil. J. Agric. Food Chem. $28,126-132$

Khan S.U. \& Ivarson K.C., (1981) Microbiological release of unextracted (bound) residues from an organic soil treated with prometryn. J. Agric. Food Chem. $29,1301-1303$

Khan S.U. \& Ivarson K.C. (1982) Release of soil bound (non extractable) residues by various physiological groups of microorganisms. J. Environ. Sci. Health Part $B$ Pestic. Food Contam. Agric. Wastes B 17, 737-749

Klein W. \& Scheunert I. (1982) Bound pesticide residues in soil, plants and food with particular emphasis on the application of nuclear techniques. In: Agrochemicals: Fate in Food and the Environment (International Atomic Energy Agency, ed.) pp. 177-205

Lichtenstein E.P., Katan J. \& Anderegg B.N. (1977) Binding of "persistent" and "non persistent" ${ }^{14} \mathrm{C}$-labeled insecticides in an agricultural soil. J. Agric. Food Chem. 25, 43-47

Mostafa I.Y., Zayed S.M.A.D., Adam Y.M. \& Attaby H.S.H. (1982) Investigations on trifluralin binding to soil and possible uptake of bound residues by plants. $J$. Environ. Sci. Health Part B Pestic. Food. Contam. Agric. Wastes 17, 265-275

Saxena A. \& Bartha R. (1983a) Modeling of the covalent attachment of chloroaniline residues to quinoidal sites of soil humus. Bull. Environ. Contam. Toxicol. 30, 485-491

Saxena A. \& Bartha R. (1983b) Binding of 3,4-dicloroaniline by humic acid and soil. Mechanism and exchangeability. Soil Sci. 136, 111-116

Scheunert I., Topp E., Schmitzer J., Klein W. \& Korte F. (1985) Formation and fate of bound residues of ${ }^{14} \mathrm{C}$ benzene and ${ }^{14} \mathrm{C}$ chlorobenzene in soil and plants. Ecotoxicol. Environ. Saf. 9, 159-170

Schiavon M. (1988) Studies of the movement and the formation of bound residues of atrazine, of its chlorinated derivatives, and of hydroxyatrazine in soil using ${ }^{14} \mathrm{C}$ ring-labeled compounds under outdoor conditions. Ecotoxicol. Environ. Saf. 15, 55- 61

Schiavon M., Jacquin F. \& Goussault C. (1977) Blocage de molécules $s$-triaziniques par la matière organique. In: Soil Organic Matter Studies Vol II. International Atomic Energy Agency, pp. 327-332

Schiavon M. \& Soulas G. (1983) Etude de la contamination des eaux de drainage et de la matière organique du sol par l'atrazine et ses dérivés de dégradation. Ministère de l'Environnement. Convention $n^{\circ} 81398$

Smith R.A., Belles W.S., Shein K.W. \& Woods W.G. (1973) The degradation of dinitramine in soil. Pestic. Biochem. Physiol. 3, 278-288

Spillner C.J., Debaun J.R. \& Menn J.J. (1979) Degradation of fenitrothion in forest soil and effects on forest soil microbes. J. Agric. Food Chem. 27, 1044-1060

U.S. Environmental Protection Agency, (1975) Fed. Regist. 40, 26802

Venkateswarlu K. \& Sethunathan N. (1979) Metabolism of carbofuran in rice straw amented and unamended rice soils J. Environ. Qual. 8, 365-368

Viswananthan R., Scheunert I., Kohli J., Klein W. \& Korte F. (1978) Longterm studies on the fate of 3,4-dichloroaniline ${ }^{14} \mathrm{C}$ in a plant-soil system under outdoor conditions. J. Environ. Sci. Health Part B Pestic. Food Contam. Agric. Wastes 13, 243-259

Worobey B.L. \& Webster B. (1982a) Hydrolytic release of tightly complexed 4-chloroaniline from soil humic acids: an analytical method. J. Agric. Food Chem. 30, 161-164

Worobey B.L. \& Webster B. (1982b) Pyrolytic release of tightly complexed 4-chloroaniline from soils and soil humic acids J. Agric. Food Chem. 30, 164-169

Yee D., Weinberger P. \& Khan S.U. (1985) Release of soil bound prometryne residues under different soil $\mathrm{pH}$ and nitrogen fertilizer regimes. Weed Sci. 33, 882-887

You I.S., Jones R.A. \& Bartha R. (1982) Evaluation of a chemically defined model for the attachment of 3,4-dichloroaniline to humus. Bull. Environ. Contam. Toxicol. 29, 476-482 